Research Article

\title{
Synthesis of Uniformly Excited Concentric Ring Arrays by the Strategy of Partial Density Tapering and the Algorithm of Differential Evolution
}

\author{
Xin-Kuan Wang $\left(\mathbb{D}\right.$, Gui-Bao Wang $\mathbb{D}^{D}$, Jianke Jia $(\mathbb{D}$, and Chao-Jun Huang \\ School of Physics and Telecommunication Engineering, Shaanxi University of Technology, Hanzhong 723001, China \\ Correspondence should be addressed to Xin-Kuan Wang; wxkuan@snut.edu.cn
}

Received 21 July 2020; Revised 10 September 2020; Accepted 29 September 2020; Published 10 October 2020

Academic Editor: Luciano Mescia

Copyright (C) 2020 Xin-Kuan Wang et al. This is an open access article distributed under the Creative Commons Attribution License, which permits unrestricted use, distribution, and reproduction in any medium, provided the original work is properly cited.

\begin{abstract}
A new strategy of density tapering called the partial density tapering (PDT) accompanied with the algorithm of differential evolution (DE) is proposed to suppress the peak sidelobe level (PSL) of uniform excited concentric ring arrays (UECRA) with isotropic elements. Through performing the PDT, a sound starting solution for DE can be generated. Then, the ring filling factor (RFF) is introduced so that the optimization of the number of elements can be transformed into the optimization of RFFs within the tapered thresholds, and thereby the real coding can be directly used with respect to the consideration of parallel encoding strategy. Finally, the UECRA featuring improved PSL performance can be obtained by limited runs of conventional DE. Several numerical instances for UECRA, with aperture sizes ranging from small to large scale, confirmed the outperformance of the proposed method.
\end{abstract}

\section{Introduction}

For the capability of achieving rotationally symmetrical beam pattern, concentric ring array (CRA) had found the applications in radar, direction finding, radio-astronomy, satellite communications, and so on [1-3]. Therefore, the synthesis for CRA had been a topic of considerable study by many investigators [1-17]. In particular, most of the previous efforts focused on the uniform excited (equal feed) CRA due to the simplified feeding network and maximized efficiency of DC-to-RF power conversion, as it is compared to the use of amplitude tapering technique. According to the literatures, we can retrieve mainly three types of methods which are developed to design concentric ring arrays (CRAs). The first is deterministic approaches (DAs) [3-9], which usually refer to emulating the continuous reference aperture distribution where a proper rule of density tapering [4-7] is employed to determine the element locations. In the past few years, several new forms of DAs are proposed to design UECRA. Angeletti et al. [3] presented a method where both locations and dimensions of the radiating elements are jointly optimized to improve the aperture efficiency of UECRA. In literature [8], Morabito and Nicolaci described a way to the optimal power synthesis of maskconstrained shaped beams. In literature [9], by utilizing the characteristics of first kind Bessel functions for representing the array factor, Kedar presented a technique for synthesis of wideband wide-scan UECRA with low sidelobe level. In all, the DAs have a fast calculation speed, which could be used for the synthesis of very large UECRA. However, this type of approaches cannot guarantee the methods which converge to the global optima.

The second type of method is the use of biologically inspired global optimization procedures such as the genetic algorithm (GA) [10] and its improved forms [11-13]. Haupt [10] earlier described the way to optimize the element placement in a UECRA to obtain the low PSL by using the GA. Then, Chen et al. [11] presented a modified real GA (MGA) which makes ring radii as the only optimal variables to suppress the PSL of UECRA. For the same issue, Jiang 
et al. [12] proposed an improved integer genetic algorithm (IIGA). In literature [13], a multiobjective GA (MOGA) is described and proved to be effective for designing the CRA, where both the half-power-beamwidth (HPBW) and PSL of the CRAs at different scanning directions are optimized.

The third type of approaches for designing UECRA is the hybrid algorithms (HAs) [2, 14-17]. By combining the advantages of some traditional optimization strategies, the deterministic procedures, the global optimization tools, etc., the use of this type of methods may achieve global optimization at a relative low-computational burden. Some typical contributions are reviewed below. Carlin et al. [2] delivered a two-step methodology which determines the sparsest auxiliary layout of concentric current rings matching a user-defined reference pattern through a Bayesian compressive sensing method and then yields the concentric ring isophoric array by strategy of density tapering. Furthermore, through splitting the synthesis of circular array into a global optimization procedure on a strongly reduced search space and a convex programming problem, Bucci and Pinchera [14] presented a hybrid approach which is proved effective for the synthesis of highdirectivity pencil beam patterns. Other notable HAs such as the method in [15] which combines a convex optimization and a determined approach together, the strategy [16] by using weighting density tapering and GA, the approach [17] integrating an improved discrete cuckoo search algorithm (IDCSA), and a cuckoo search-invasive weed algorithm (CSIWA) [17].

Of all the previous efforts, the biological-based methods such as GA [10-12] and MOGA [13] have the advantages of global optimization, but are only suitable for dealing with small arrays. Actually, the reports for synthesizing UECRA of large scale by a limited runs of GA [10] are proved to be time consuming, and thereby local minima is resulted. The global optima may be obtained by many runs of the method, which means the needing of huge computational efforts. According to the authors' opinion, improving the quality of the initial solution is a reasonable way to speed up the convergence speed of the stochastic algorithms. In view of this point and with the purpose of suppressing the PSL of UECRA with isotropic elements, a new strategy of density tapering, named partial density tapering (PDT), is proposed in this paper. The idea of PDT is derived from the technique of amplitude tapering, typical as the solution when performing Taylor tapering to achieve an ultralow sidelobe array. The amplitudes of element excitations show a tapered distribution from the center of array to the outermost. In particular, for the elements located near the center of the array, the normalized excited amplitudes are almost equal and close to 1 . For this reason, we first divide the aperture of UECRA into $M$ concentric rings, where a total of $m_{0}$ rings $\left(m_{0}\right.$ is the nearest integer less than or equal to $M / 2$ ) close to the center of the array are set to be equal-spaced, and the interspacings of remaining $\left(M-m_{0}\right)$ rings are set to be sequentially increased from the inside to the outside of the array. Then, we initialize the element distribution of $m_{0}$ inner rings to be fully populated, while the ring filling factor (RFF, defined as the ratio of the current number of elements of a ring to the number of elements when the ring is fully populated) of the rest rings are constrained by a proper tapered thresholds. For this reason, we name the strategy as PDT.

After the implementation of PDT, both the ring radii and RFF of the outer $\left(M-m_{0}\right)$ rings are optimized by a conventional algorithm of DE; thus, we call the method PDTDE. Unlike the IIGA [12] where a parallel encoding strategy is adopted, this method transforms the number of elements on a ring into the value of RFF between $(0,1)$, and thereby real coding can be directly used in the optimization procedure.

The paper is organized as follows. Section 2 describes the formulation of the proposed method. Section 3 provides $a$ set of numerical examples to validate the effectiveness of the PDT-DE. Finally, the conclusion of this work is summarized in Section 4.

\section{Formulation of the Proposed Method}

For a single element at the center, the array factor of a UECRA with isotropic elements can be represented by

$$
F(u, v)=1+\sum_{m=1}^{M} \sum_{n=1}^{N_{m}} e^{j k r_{m}\left(u \cos \phi_{m n}+v \sin \phi_{m n}\right)}
$$

where $r_{m}(m=1,2, \ldots, M)$ is the radius of ring $m$ that consists of $N_{m}$ isotropic elements. $M$ denotes the total number of rings and $k$ is the wave number. $\phi_{m n}$ depicts the angular location of element $n$ in ring $m$, which is expressed by $\phi_{m n}=2 \pi(n-1) / N_{m} . u=\sin \theta \cos \phi$ and $v=\sin \theta \sin \phi, \theta$ and $\phi$ are the elevation and azimuth angles in spherical coordinates.

For a given ring, we specify that the minimum arc length interval between adjacent elements is $0.5 \lambda$ ( $\lambda$ denotes the wavelength). Then, the maximum number of elements that can be filled in ring $m$ (we mean that the ring $m$ is fully populated) is given by

$$
N_{m}^{\max }=\operatorname{int}\left(\frac{2 \pi r_{m}}{0.5 \lambda}\right), \quad m=1,2, \ldots, M,
$$

where int $(A)$ denotes the function that rounds the value of $A$ to the nearest integer less than or equal to $A$.

Suppose a UECRA consisting of $M$ rings is synthesized using the PDT-DE within a circular aperture of radius $R$; thus, the detailed procedures of the proposed method are depicted as follows.

2.1. Aperture Division. Divide the aperture into $M$ concentric rings, where both $R$ and $M$ should conform to the relationship:

$$
\left\{\begin{array}{l}
\epsilon=R-M \cdot d_{0}, \\
\text { s.t. } \varepsilon \geq 0.5 \lambda, \quad d_{0} \in(0.5 \lambda, 0.6 \lambda),
\end{array}\right.
$$

where $\epsilon$ is the difference of the radii between aperture radius and the outermost ring when we assume that all the rings are spaced at $d_{0}$. For convenience, rewrite $d_{0}$ as 


$$
d_{0}=(0.5+0.1 \sigma) \lambda,
$$

where $\sigma$ is a randomly selected value from interval $(0,1)$. Furthermore, we force $m_{0}=\operatorname{int}(M / 2)$, and the radii of $m_{0}$ rings close to the center of the aperture can be expressed by

$$
r_{m}=m \cdot d_{0}, \quad m=1,2, \ldots, m_{0} .
$$

For the rest $\left(M-m_{0}\right)$ rings located away from the center of the aperture, the radii are specified by

$$
\left\{\begin{array}{l}
r_{m_{0}+q}=\left(m_{0}+q\right) \cdot d_{0}+\Delta d_{q}, \\
\text { s.t. } 0<\Delta d_{q}<\epsilon, \quad q=1,2, \ldots M-m_{0} .
\end{array}\right.
$$

For convenience, a vector is introduced to describe the collection of $\Delta d_{q}, \quad q=1,2, \ldots, M-m_{0}$, as

$$
\Delta \mathbf{d}=\left\{\Delta d_{1}, \Delta d_{2}, \ldots, \Delta d_{M-m_{0}}\right\} .
$$

Let $\Delta r_{m}=r_{m}-r_{m-1}$, which means the difference of radii between $m$ th and $(m-1)$ th rings; then, we have

$$
\begin{cases}\Delta r_{m}=d_{0}, & \text { for } 2 \leq m \leq m_{0}, \\ \Delta r_{m}>d_{0}, & \text { for } m_{0}<m \leq M .\end{cases}
$$

According to equations (6) and (7) and in order to meet the requirement of density tapering, the variables in vector $\Delta \mathbf{d}$ need to be arranged in ascending order. To determine $\Delta \mathbf{d}$, a new vector is defined as

$$
\boldsymbol{\tau}=\left\{\tau_{1}, \tau_{2}, \ldots, \tau_{M-m_{0}}, \tau_{1+M-m_{0}}, \tau_{2+M-m_{0}}, \ldots, \tau_{L+M-m_{0}}\right\},
$$

where $L$ is an integer that is randomly selected from 0 to 5 and $\tau_{i} \quad\left(1 \leq i \leq L+M-m_{0}\right)$ is the random number uniformly distributed within $(0,1)$. Through sorting $\left\{\tau_{i}\right\}$ in ascending order, we get a distinct vector:

$$
\boldsymbol{\tau}^{\prime}=\left\{\tau_{1}^{\prime}, \tau_{2}^{\prime}, \ldots \tau_{M-m_{0}}^{\prime}, \tau_{1+M-m_{0}}^{\prime}, \tau_{2+M-m_{0}}^{\prime}, \ldots, \tau_{L+M-m_{0}}^{\prime}\right\} .
$$

Based on (3) and (10), we divide $\epsilon$ into $\left(L+M-m_{0}\right)$ unequal parts, and the value of each part is described by

$$
\delta_{i}=\frac{\epsilon}{\sum_{i=1}^{M-m_{0}+L} \tau_{i}^{\prime}} \cdot \tau_{i}^{\prime}, \quad i=1,2, \ldots, L+M-m_{0} .
$$

Let a new vector to record (11) be

$$
\boldsymbol{\delta}=\left\{\delta_{1}, \delta_{2}, \ldots, \delta_{L}, \delta_{L+1}, \ldots, \delta_{L+M-m_{0}}\right\} .
$$

Previous derivation shows that $\delta_{i} \quad(i=1,2, \ldots, L+$ $\left.M-m_{0}\right)$ increases sequentially with the increment of subscripts. Furthermore, we split $\delta$ into two vectors (denoted as $\delta^{\prime}$ and $\delta^{\prime \prime}$ ) and record them as

$$
\begin{aligned}
\boldsymbol{\delta}^{\prime} & =\left\{\delta_{1}, \delta_{2}, \ldots, \delta_{L}\right\}, \\
\boldsymbol{\delta}^{\prime \prime} & =\left\{\delta_{L+1}, \ldots, \delta_{L+M-m_{0}}\right\} .
\end{aligned}
$$

Let $\Delta \mathbf{d}=\delta^{\prime \prime}$; therefore, $\Delta d_{q}=\delta_{L+q} \quad(q=1,2, \ldots, M$ $\left.-m_{0}\right)$.
After the implementation of equations (3)-(14), all the ring radii of UECRA are determined. For rings from 1th to $m_{0}$ th, the spacings are equal to $d_{0}$. Differently, for rings from $\left(m_{0}+1\right)$ th to $M$ th, the ring intervals are greater than $d_{0}$, and they are gradually increased from the inside to the outside of the array.

The use of vector $\delta^{\prime}$ is to make a slight adjustment to the radius of outermost ring so that $r_{M} \leq R$. In particular, when $L=0, \delta^{\prime}$ becomes to an empty set; thus, we have $r_{M}=R$, or else $r_{M}<R$. The operation helps to meet the design targets under possible smallest aperture radius.

2.2. Initialize the Element Distribution. Firstly, the rings from 1th to $m_{0}$ th are fully populated, and the number of elements for each ring is determined by (2). The total number of elements in the full structure is recorded as $\sum_{m=1}^{m_{0}} N_{m}^{\max }$. For the rest rings, the ring filling factors (RFFs) are expressed by

$$
f_{q}=\frac{N_{m_{0}+q}}{N_{m_{0}+q}^{\max }} \times 100 \%, \quad q=1,2, \ldots, M-m_{0},
$$

where $N_{m_{0}+q}$ denotes the current number of elements in ring $m_{0}+q$ and $N_{m_{0}+q}^{\max }$ is determined by (2). We set a new vector to describe (15) as

$$
\mathbf{f}_{\mathrm{RFF}}=\left\{f_{1}, f_{2}, f_{3}, \ldots, f_{M-m_{0}}\right\},
$$

where $f_{q} \quad\left(q=1,2, \ldots, M-m_{0}\right)$ are bounded by

$$
f_{q}^{\min }<f_{q}<f_{q}^{\max }, \quad q=1,2, \ldots, M-m_{0} .
$$

For convenience, the collections of $\left\{f_{q}^{\min }\right\}$ and $\left\{f_{q}^{\max }\right\}$ are, respectively, labeled as

$$
\begin{aligned}
\mathbf{f}_{\mathrm{RFF}}^{\min } & =\left\{f_{1}^{\min }, f_{2}^{\min }, f_{3}^{\min }, \ldots, f_{M-m_{0}}^{\min }\right\}, \\
\mathbf{f}_{\mathrm{RFF}}^{\max } & =\left\{f_{1}^{\max }, f_{2}^{\max }, f_{3}^{\max }, \ldots, f_{M-m_{0}}^{\max }\right\} .
\end{aligned}
$$

Both $\mathbf{f}_{\mathrm{RFF}}^{\max }$ and $\mathbf{f}_{\mathrm{RFF}}^{\min }$ constitute the upper and lower filling thresholds for $\mathbf{f}_{\mathrm{RFF}}$. In order to meet the requirement of density tapering, the variables of the two vectors should be given in descending order, respectively. In more detail, we set $f_{q}^{\min }\left(q=1,2, \ldots, M-m_{0}\right)$ to be arranged in descending order within the interval $[0.25,0.8]$, and accordingly $f_{q}^{\max } \quad\left(q=1,2, \ldots, M-m_{0}\right)$ are determined by

$$
\begin{cases}f_{q}^{\max }=f_{q}^{\min }+\eta_{1}, & \text { when } f_{q}^{\min } \geq 0.75, \\ f_{q}^{\max }=f_{q}^{\min }+\eta_{2}, & \text { otherwise, }\end{cases}
$$

where we specify $\eta_{1} \in[0.1,0.2], \eta_{2} \in[0.1,0.25]$ so that the RFFs for rings from $\left(m_{0}+1\right)$ th to $M$ th are less than 1.0. Not only that, the curves of RFF thresholds need to have a relatively gentle tapering to expand the solution space and enhance the capability of global convergence of the algorithm. Therefore, a scope $0.05 \leq \eta_{2}-\eta_{1} \leq 0.1$ is recommended. However, the parameters of $\eta_{1}$ and $\eta_{2}$ are not strictly limited, as long as the values ensure that the RFFs are less than 1.0 and both the RFF thresholds satisfies a proper tapering distribution. 


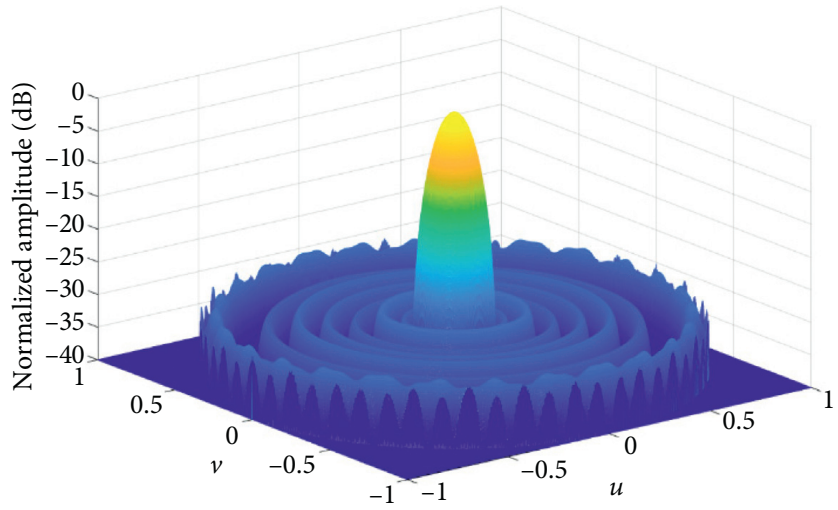

Figure 1: 3D pattern of the UECRA when $N=142$.

2.3. DE Optimizing. The individual vector of the population is constituted by $\sigma, \tau$, and $\mathbf{f}_{\mathrm{RFF}}$, which can be described as

$$
\mathbf{X}=\left\{\sigma, \tau, \mathbf{f}_{\mathbf{R F F}}\right\}
$$

with

$$
\left\{\begin{array}{l}
0<\sigma<1, \\
0<\tau_{i}<1, \quad i=1,2, \ldots, L+M-m_{0}, \\
f_{q}^{\min }<f_{q}<f_{q}^{\max }, \quad q=1,2, \ldots, M-m_{0} .
\end{array}\right.
$$

Both $\sigma$ and $\tau$ are used to adjust the ring radii of UECRA, while $\mathbf{f}_{\mathrm{RFF}}$ determines the number of elements that can be filled in different rings. Suppose $\tau_{\max }=\{1,1, \ldots, 1\}$ and $\tau_{\text {min }}=\{0,0, \ldots, 0\}$ and both the two vectors have the same length with $\tau$. Correspondingly, the upper and lower thresholds for $\mathbf{X}$ can be expressed by

$$
\left\{\begin{array}{l}
\mathbf{X}_{\max }=\left\{0, \boldsymbol{\tau}_{\max }, \mathbf{f}_{\mathrm{RFF}}^{\max }\right\}, \\
\mathbf{X}_{\min }=\left\{1, \boldsymbol{\tau}_{\min }, \mathbf{f}_{\mathrm{RFF}}^{\min }\right\} .
\end{array}\right.
$$

According to (23), more individuals of DE are generated through the equation:

$$
\mathbf{X}=\mathbf{X}_{\min }+\mathbf{R}_{\mathrm{and}} \cdot\left(\mathbf{X}_{\max }-\mathbf{X}_{\min }\right),
$$

where $\mathbf{R}_{\text {and }}$ is a random vector that has the same length with $\mathbf{X}$, and the variables in $\mathbf{R}_{\text {and }}$ are the random numbers uniformly distributed within $(0,1)$. The fitness function are defined as

$$
\min \text { PSL }=\zeta(\mathbf{X})
$$

where $\zeta(\mathbf{X})$ denotes the UECRA that can be obtained with minimum PSL (the fitness value). After the whole population is formed, the individuals are optimized by a conventional DE and evaluated by the fitness function described in (25). Simultaneously, the elite strategy is adopted so that the UECRA featuring minimum PSL is retained.

\section{Numerical Examples}

The proposed method is applied to the design cases similar to that taken from $[10-12,16]$, where a single element is existed at the center of UECRA. The parameters involved in the method, including the population size, the maximum number of iterations in each generation, and crossover probability, as well as the scaling factor, are equal to 50, 20, 0.9 , and 0.8 , respectively.

In the first case, a circular aperture with radius of $4.5 \lambda$ is considered, among which the array consists of 7 rings and 142 elements. According to the proposed method, the inner three rings are fully populated. For the four outer rings, we renumbered them from inside to the outside as $1 \#, 2 \#, 3 \#$, and 4\#. Theoretically, the upper and lower thresholds for filling factors of the four rings should be prespecified, as are described by (18)-(20). However, since the total number of elements is given in advance, only the RFF thresholds of $1 \# \sim 3 \#$ rings need to be predetermined. The number of elements in the $4 \#$ ring is equal to the difference between 141 and the total number of elements included in the remaining six rings. We specify the lower and upper RFF thresholds as $[0.8,0.65,0.5]$ and $[1.0,0.9,0.75]$, respectively. The obtained array after 50 runs of the PDT-DE has the PSL of $-29.95 \mathrm{~dB}$, which is $2.13 \mathrm{~dB}$ lower than the value obtained by the GA [10] and $1.62 \mathrm{~dB}$ lower than the report obtained by the MGA [11]. Not only that, compared with the report in [10], the aperture radius is reduced from $4.7 \lambda$ to $4.26 \lambda$, and the directivity (denoted as $D$ ) is lost by $0.7 \mathrm{~dB}$.

Similarly, when we increase the total number of elements in UECRA to 183 and the number of rings to 8 , the PSL of synthesized array is equal to $-27.86 \mathrm{~dB}$, which shows a PSL reduction of $2.28 \mathrm{~dB}$ with a little sacrifice at directivity, as it is compared to the value demonstrated by GA [10]. Described in Figures 1 and 2 are the 3D pattern of previous two instances, where $N$ denotes the total number of elements. In addition, for the case when $N=201$, the best array after 50 runs of the PDT-DE has a PSL equal to $-30.34 \mathrm{~dB}$. The performance of the array shows a PSL suppression of $1.21 \mathrm{~dB}$ compared to the value obtained by the MGA [11] and a PSL suppression of $6.13 \mathrm{~dB}$ compared to that demonstrated by the IFT-DE [18]. More detailed results, including the ring radii and the number of elements in each ring, are given in Table 1.

In the fourth case, we consider a circular array with aperture radius of $15 \lambda$, and the total number of elements of 1302. Then, we set the maximum number of runs equal to 20 to alleviate the computational burden. The best fitness value 


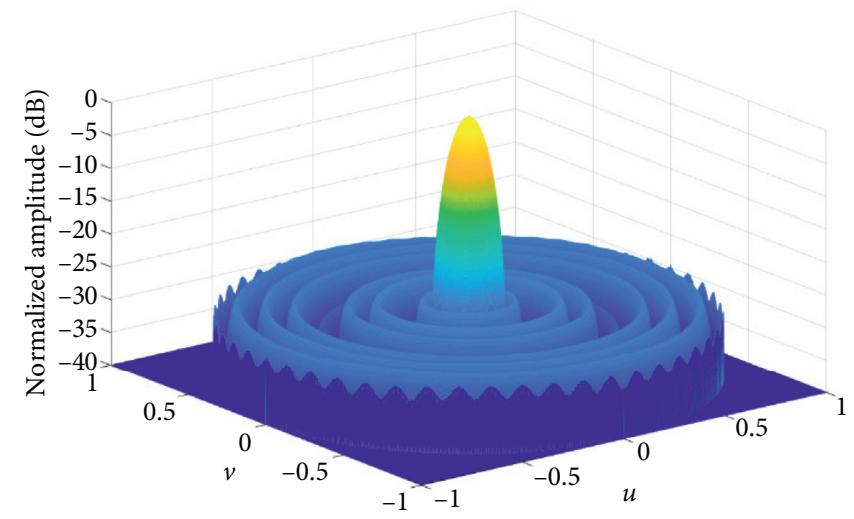

Figure 2: 3D pattern of the UECRA when $N=183$.

TABLE 1: Synthesis results obtained by PDT-DE for the cases $N=142,183$, and 201.

\begin{tabular}{|c|c|c|c|c|c|c|c|c|c|c|c|c|}
\hline$R(\lambda)$ & $N$ & PSL (dB) & $D(\mathrm{~dB})$ & $m$ & 1 & 2 & 3 & 4 & 5 & 6 & 7 & 8 \\
\hline \multirow{2}{*}{4.26} & \multirow{2}{*}{142} & \multirow{2}{*}{-29.95} & \multirow{2}{*}{28.19} & $r_{m}(\lambda)$ & 0.5 & 1.0 & 1.5 & 2.06 & 2.68 & 3.45 & 4.26 & - \\
\hline & & & & $N_{m}$ & 6 & 12 & 18 & 21 & 29 & 26 & 29 & - \\
\hline \multirow{2}{*}{4.5} & \multirow{2}{*}{183} & \multirow{2}{*}{-27.86} & \multirow{2}{*}{28.39} & $r_{m}(\lambda)$ & 0.5 & 1.0 & 1.5 & 2.0 & 2.5 & 3.07 & 3.68 & 4.5 \\
\hline & & & & $N_{m}$ & 6 & 12 & 18 & 25 & 25 & 28 & 25 & 43 \\
\hline \multirow{2}{*}{5.0} & \multirow{2}{*}{201} & \multirow{2}{*}{-30.34} & \multirow{2}{*}{29.30} & $r_{m}(\lambda)$ & 0.5 & 1.0 & 1.5 & 2.0 & 2.65 & 3.36 & 4.11 & 5.0 \\
\hline & & & & $N_{m}$ & 6 & 12 & 18 & 25 & 33 & 35 & 31 & 40 \\
\hline
\end{tabular}

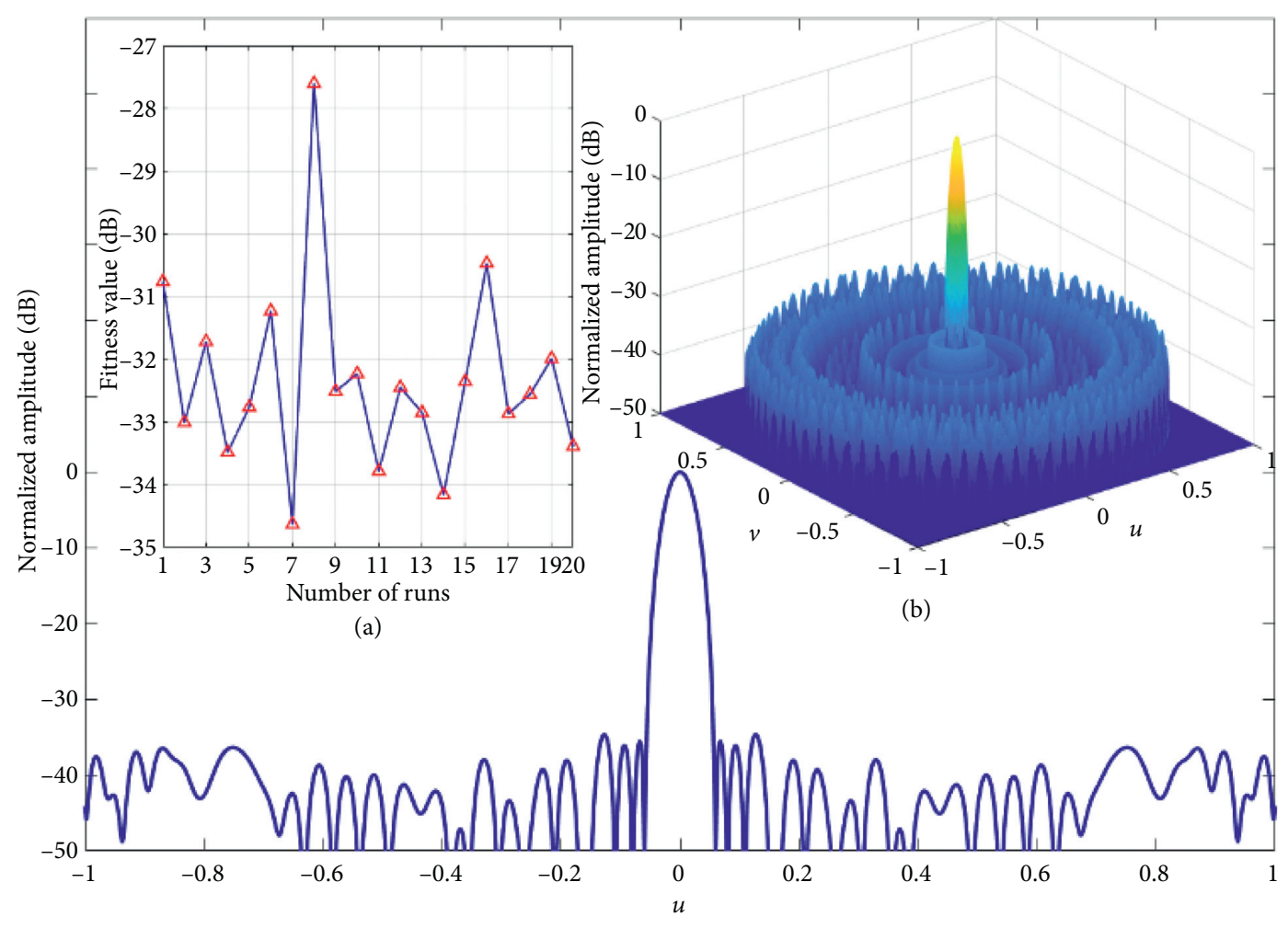

- u-cut pattern

(c)

FIgUre 3: (a) Convergence of fitness value, (b) 3D pattern, and (c) u-cut.

is obtained at the 7 th run, where the array has the PSL of $-34.63 \mathrm{~dB}$, as shown in Figure 3(a). The value is $1.67 \mathrm{~dB}$ lower than the report demonstrated by IIGA [12] with a
$0.11 \mathrm{~dB}$ loss of directivity. Correspondingly, a reduction of aperture radius about $0.36 \lambda$ is resulted. Figures 3(b) and 3(c) describe the $3 \mathrm{D}$ and $\mathrm{u}$-cut pattern of the array, respectively. 


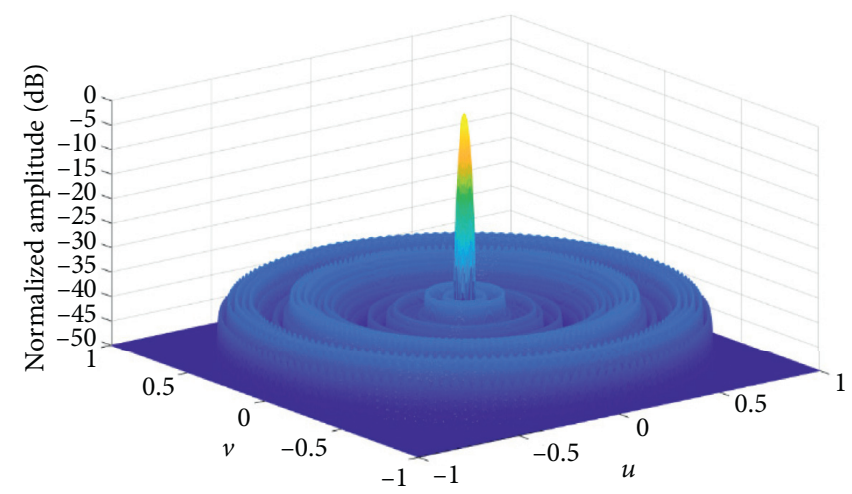

Figure 4: 3D pattern of the UECRA when $N=2256$.

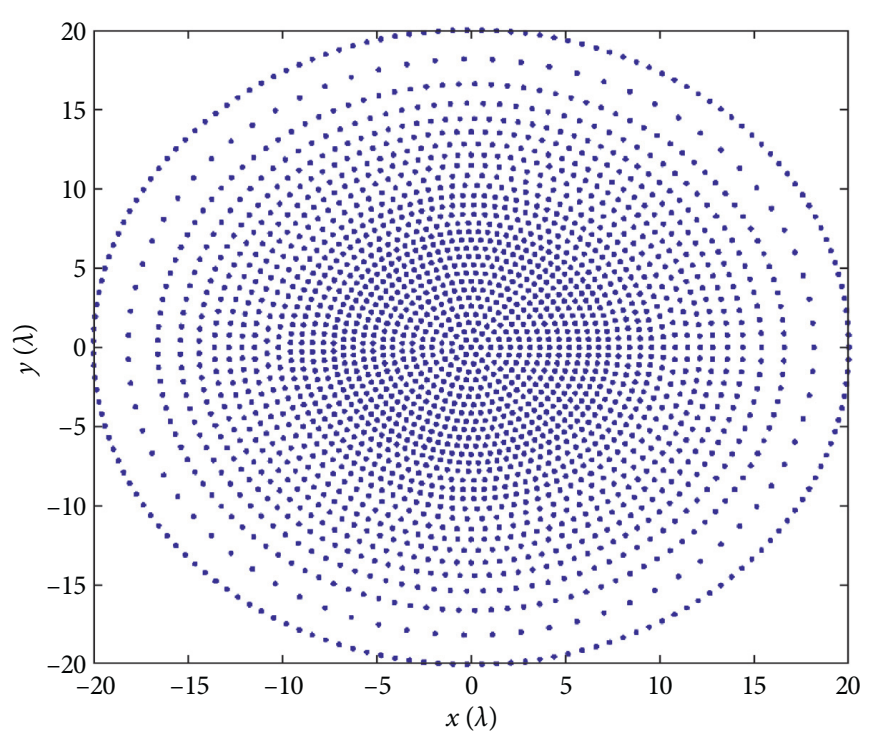

FIgURE 5: Element distribution of the UECRA when $N=2256$.

Furthermore, the method is extended for the synthesis of large-scale UECRA within an aperture radius of $20 \lambda$, among which the array consist of 2256 elements and 29 rings. The best array by 20 runs of the PDT-DE has the PSL of $-36.33 \mathrm{~dB}$ and directivity of $39.54 \mathrm{~dB}$, which show about $2.25 \mathrm{~dB}$ PSL reduction with only $0.12 \mathrm{~dB}$ loss of directivity, as compared to the reports obtained by HS [16].

Figures 4 and 5 depict the 3D pattern and element distribution within the aperture of the UECRA, respectively. For the same array, the ring radii and the number of elements in each ring are tabulated in Table 2. More synthesis results by using different methods are compared in Table 3, where we can conclude that the PDT-DE has the advantages in PSL suppression of UECRA with respect to other methods, and the PSL performance of the array become more impressive for large scale UECRA by using the proposed method. All the numerical results mentioned above are obtained by using a PC equipped with an $8 \mathrm{~GB}$ RAM, and the Intel I7-6700 processor that operates at $3.4 \mathrm{GHz}$.
TABLE 2: Results of ring radii $\left(r_{m}\right)$ and number of elements $\left(N_{m}\right)$ for the UECRA including 2256 elements.

\begin{tabular}{lcccccccc}
\hline$m$ & $r_{m}$ & $N_{m}$ & $m$ & $r_{m}$ & $N_{m}$ & $m$ & $r_{m}$ & $N_{m}$ \\
\hline 1 & 0.52 & 6 & 11 & 5.72 & 71 & 21 & 11.46 & 96 \\
2 & 1.04 & 13 & 12 & 6.24 & 78 & 22 & 12.13 & 108 \\
3 & 1.56 & 19 & 13 & 6.76 & 84 & 23 & 12.81 & 106 \\
4 & 2.08 & 26 & 14 & 7.28 & 91 & 24 & 13.58 & 112 \\
5 & 2.60 & 32 & 15 & 7.82 & 87 & 25 & 14.40 & 117 \\
6 & 3.12 & 39 & 16 & 8.39 & 89 & 26 & 15.38 & 111 \\
7 & 3.64 & 45 & 17 & 8.97 & 101 & 27 & 16.59 & 121 \\
8 & 4.16 & 52 & 18 & 9.56 & 105 & 28 & 18.16 & 75 \\
9 & 4.68 & 58 & 19 & 10.19 & 98 & 29 & 20.0 & 163 \\
10 & 5.20 & 65 & 20 & 10.81 & 87 & - & - & - \\
\hline
\end{tabular}

TABLE 3: Comparative results by using different methods.

\begin{tabular}{lccccc}
\hline$R(\lambda)$ & \multirow{2}{*}{$N$} & Methods & PSL (dB) & D (dB) & $\begin{array}{c}\text { 3dB beamwidth } \\
(\Delta u)\end{array}$ \\
\hline 4.70 & \multirow{3}{*}{142} & GA [10] & -27.82 & 28.89 & - \\
4.70 & MGA [11] & -28.33 & - & - \\
4.26 & & PDT-DE & -29.95 & 28.19 & 0.128 \\
\hline 4.5 & \multirow{2}{*}{183} & GA [10] & -25.58 & 28.5 & - \\
4.5 & & PDT-DE & -27.86 & 28.39 & 0.115 \\
\hline 4.98 & \multirow{2}{*}{201} & MGA [11] & -29.13 & - & - \\
5.0 & IFT-DE [18] & -24.21 & - & - \\
5.0 & & PDT-DE & -30.34 & 29.30 & 0.108 \\
\hline 10 & \multirow{2}{*}{623} & IIGA [12] & -32.44 & 34.75 & 0.0569 \\
10 & & PDT-DE & -33.60 & 34.33 & 0.06 \\
\hline 15 & \multirow{2}{*}{1302} & IIGA [12] & -32.96 & 37.49 & 0.0388 \\
14.64 & & PDT-DE & -34.63 & 37.38 & 0.04 \\
\hline 20 & \multirow{2}{*}{2256} & HS [16] & -34.08 & 39.66 & 0.0296 \\
20 & & PDT-DE & -36.33 & 39.54 & 0.032 \\
\hline 25 & \multirow{2}{*}{3465} & IIGA [12] & -33.64 & 41.37 & 0.0236 \\
24.78 & & PDT-DE & -35.58 & 41.19 & 0.024 \\
\hline
\end{tabular}

\section{Conclusion}

The proposed method is capable of achieving UECRA with lower PSL than the published reports, and the loss of directivity can be neglected. Not only that, it has the advantage 
of easy encoding with respect to IIGA. Therefore, the PDTDE provides an effective way for the synthesis of UECRA with aperture sizes ranging from small to large scale. Furthermore, by some modifications, the method can also be extended for the sidelobe suppression of sparse linear arrays.

\section{Data Availability}

The data used to support the findings of this study are available from the corresponding author upon request.

\section{Conflicts of Interest}

The authors declare that they have no conflicts of interest.

\section{Acknowledgments}

This work was supported in part by the National Natural Science Foundation of China under Grant nos. 61601272, 61772398, and 61972239, Key Research and Development Program Projects of Shaanxi Province under Grant no. 2019SF-257, Scientific Research Plan of Shaanxi Provincial Education Department under Grant no. 19JK0175, and Scientific Research Plan of Shaanxi University of Technology under Grant no. SLGRCQD2009.

\section{References}

[1] H. EI-Kamchouchi, "Ultra-low-sidelobe-level concentric-ringarray pattern synthesis using Bessel neural networks," IEEE Antennas and Propagation Magazine, vol. 52, no. 4, pp. 102105, 2010.

[2] M. Carlin, G. Oliveri, and A. Massa, "Hybrid BCS-deterministic approach for sparse concentric ring isophoric arrays," IEEE Transactions on Antennas and Propagation, vol. 63, no. 1, pp. 378-383, 2015.

[3] P. Angeletti, G. Toso, and G. Ruggerini, "Array antennas with jointly optimized elements positions and dimensions part II: planar circular arrays," IEEE Transactions on Antennas and Propagation, vol. 62, no. 4, pp. 1627-1639, 2014.

[4] R. Willey, "Space tapaering of linear and planar arrays," IRE Transactions on Antennas and Propagation, vol. 10, no. 4, pp. 369-377, 1962.

[5] T. A. Milligan, "Space-tapered circular (ring) array," IEEE Antennas and Propagation Magazine, vol. 46, no. 3, pp. 70-73, 2004.

[6] O. M. Bucci and S. Perna, "A deterministic two dimensional density taper approach for fast design of uniform amplitude pencil beams arrays," IEEE Transactions on Antennas and Propagation, vol. 59, no. 8, pp. 2852-2861, 2011.

[7] Y. Jiang and S. Zhang, "An innovative strategy for synthesis of uniformly weighted circular aperture antenna array based on the weighting density method," IEEE Antennas and Wireless Propagation Letters, vol. 12, pp. 725-728, 2013.

[8] A. F. Morabito and P. G. Nicolaci, "Optimal synthesis of shaped beams through concentric ring isophoric sparse arrays," IEEE Antennas and Wireless Propagation Letters, vol. 16, pp. 979-982, 2017.

[9] A. Kedar, "Deterministic synthesis of wide scanning sparse concentric ring antenna arrays," IEEE Transactions on Antennas and Propagation, vol. 67, no. 12, pp. 7387-7395, 2019.
[10] R. L. Haupt, "Optimized element spacing for low sidelobe concentric ring arrays," IEEE Transactions on Antennas and Propagation, vol. 56, no. 1, pp. 266-268, 2008.

[11] K.-S. Chen, Y.-Y. Zhu, X.-L. Ni, and H. Chen, "Low sidelobe sparse concentric ring arrays optimization using modified GA," International Journal of Antennas and Propagation, vol. 2015, pp. 1-5, 2015.

[12] Y. Jiang, S. Zhang, Q. Guo, and M. Li, "Synthesis of uniformly excited concentric ring arrays using the improved integer GA," IEEE Antennas and Wireless Propagation Letters, vol. 15, pp. 1124-1127, 2016.

[13] D. Bianchi, S. Genovesi, and A. Monorchio, "Constrained pareto optimization of wide band and steerable concentric ring arrays," IEEE Transactions on Antennas and Propagation, vol. 60, no. 7, pp. 3195-3204, 2012.

[14] O. M. Bucci and D. Pinchera, "A generalized hybrid approach for the synthesis of uniform amplitude pencil beam ringarrays," IEEE Transactions on Antennas and Propagation, vol. 60, no. 1, pp. 174-183, 2012.

[15] X. Zhao, Q. Yang, and Y. Zhang, "A hybrid method for the optimal synthesis of 3-D patterns of sparse concentric ring arrays," IEEE Transactions on Antennas and Propagation, vol. 64, no. 2, pp. 515-524, 2016.

[16] Y. Jiang, S. Zhang, Q. Guo, and X. Luan, "A hybrid strategy based on weighting density and genetic algorithm for the synthesis of uniformly weighted concentric ring arrays," IEEE Antennas and Wireless Propagation Letters, vol. 16, pp. 186189, 2017.

[17] G. Sun, Y. Liu, Z. Chen, S. Liang, A. Wang, and Y. Zhang, "Radiation beam pattern synthesis of concentric circular antenna arrays using hybrid approach based on cuckoo search," IEEE Transactions on Antennas and Propagation, vol. 66, no. 9, pp. 4563-4576, 2018.

[18] L. Wang, X.-K. Wang, G. Wang, and J.-K. Jia, “A two-step method for the low-sidelobe synthesis of uniform amplitude planar sparse arrays," Progress In Electromagnetics Research $M$, vol. 86, pp. 153-162, 2019. 